\title{
Tanzanian mushrooms and their uses 3. Termitomyces singidensis, sp. nov.
}

\author{
TIINA SAARIMÄKI, MARJA HÄRKÖNEN and LEONARD MWASUMBI
}

\begin{abstract}
SAARIMÄKI, T., HÄRKÖNEN, M. \& MWASUMBI, L. 1994: Tanzanian mushrooms and their uses 3. Termitomyces singidensis, sp. nov. - Karstenia 34:13-20. ISSN 04533402

A new Termitomyces (Agaricales), T. singidensis Saarim. \& Härk., is described and illustrated. Found in several localities near Singida town in central Tanzania, East Africa, it is collected for home consumption and offered for sale in local markets and on roadsides. Its resemblance to $T$. lanatus R.Heim, $T$. magoyensis Otieno, T. mammiformis R.Heim, and $T$. schimperi (Pat.) R.Heim is discussed.
\end{abstract}

Kew words: Africa, Agaricales, ethnomycology, Tanzania, taxonomy, Termitomyces singidensis

Tiina Saarimäki and Marja Härkönen, Department of Botany, P.O. Box 7, FIN-00014 University of Helsinki, Finland

Leonard Mwasumbi, Department of Botany, University of Dar es Salaam, P.O. Box 35060, Dar es Salaam, Tanzania

\section{Introduction}

Termitomyces R.Heim, a genus of Agaricales, occurs in the tropics of Asia', Africa, and the South Pacific (Singer 1986). Distinctive macroscopic characteristics of the genus are an obligate association with termite nests, usually a long pseudorrhiza, and a differentiated perforatorium or umbo. Most species are highly valued for food. From East Africa, Otieno (1964) has reported 15 species and Pegler \& Rayner (1969) and Pegler (1977), 11 species. From Malawi Morris (1986) has reported eight species and Piearce (1987), 11 species from Zambia. Seven species were reported from South Africa by van der Westhuizen \& Eicker (1990) and one from Namibia (van der Westhuizen \& Eicker 1991).

This article is a further contribution, consisting of a series of studies dealing with edible mushrooms of Tanzania. The research area and methods, as well as some of the results, were introduced in our previous articles (Härkönen 1992, Härkönen et al. 1993a-d).

\section{Material and methods}

All ten specimens studied were collected in central Tanzania, one in Iringa and nine in Singida District. In this text, collecting sites are according to Polhill (1988) and Leistner \& Morris (1976). All specimens are deposited at the Botanical Museum of the University of Helsinki $(\mathrm{H})$ and some representative specimens in the Herbarium of Dar es Salaam (DSM). All the authors have taken part in the collecting, Härkönen being the director responsible of the project. The microscopic examination and measurements were done and the text was written mostly by Saarimäki. Mwasumbi assisted with the section "Ecology", and Härkönen in preparing the final version of the article. The photographs are by the first two authors.

Hand sections for microscopic examination were mounted in different chemical reagents. Congo Red and Cotton Blue were used for measuring basidia, basidiospores, cheilo- and pleurocystidia, hyphae and sphaerocysts, whereas Meltzer's Reagent was for testing the chemical reaction of basidiospores.

Spores were studied from small segments of gills or from spore prints, but for one specimen no mature spores could be obtained. In the description the range of spore size and the arithmetical mean is based on 30 fully developed spores of each specimen. $\mathrm{Q}=$ quotient of the mean spore length and the mean spore width (L/W ratio). 

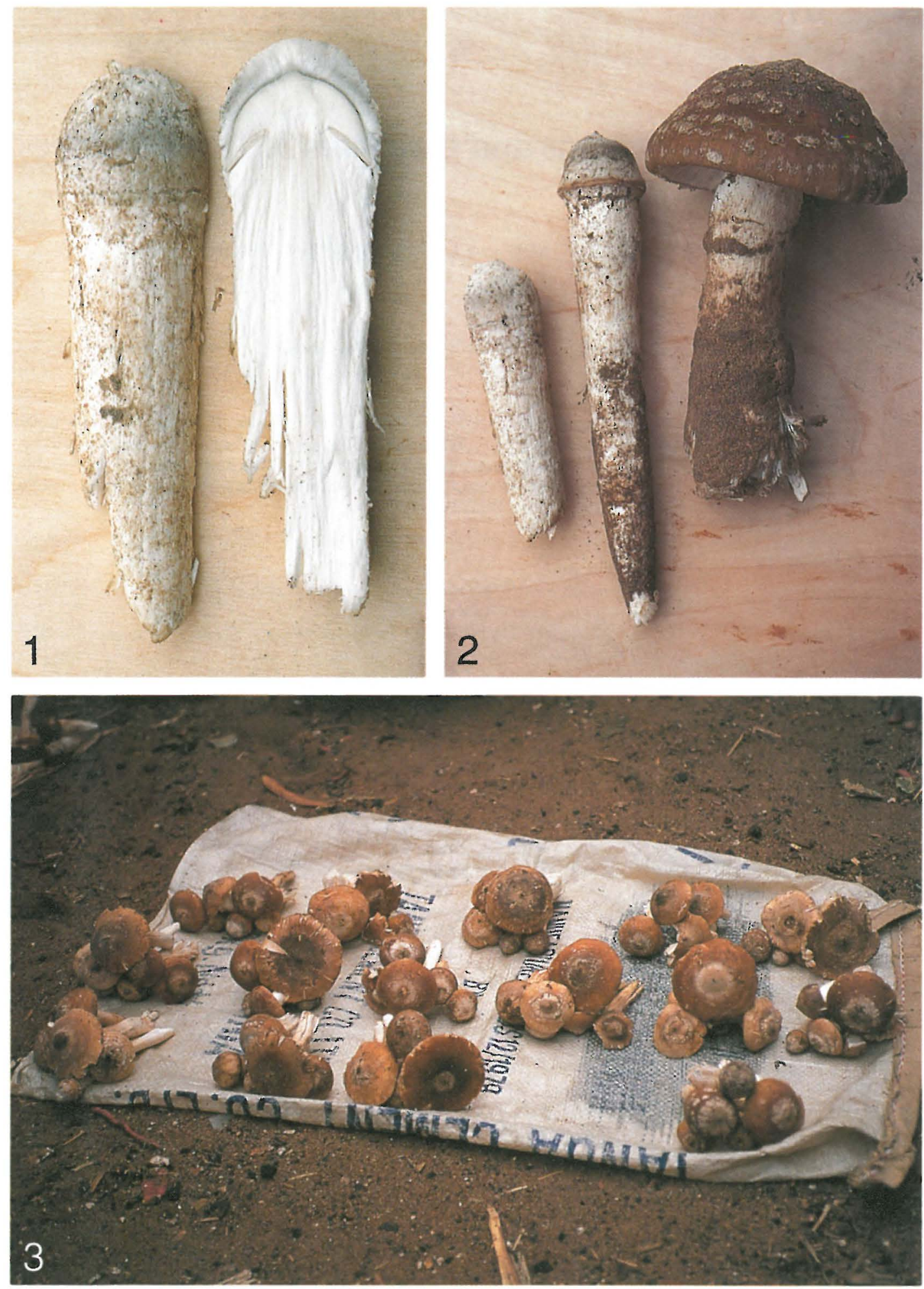

Figs. 1-3. Fruit bodies of $T$. singidensis. - 1: A young cap covered with a thick, fluffy veil (type). - 2 : Range of varying stages (type). - 3: Typical small heaps of mushrooms offered for sale at market in Singida, central Tanzania. 

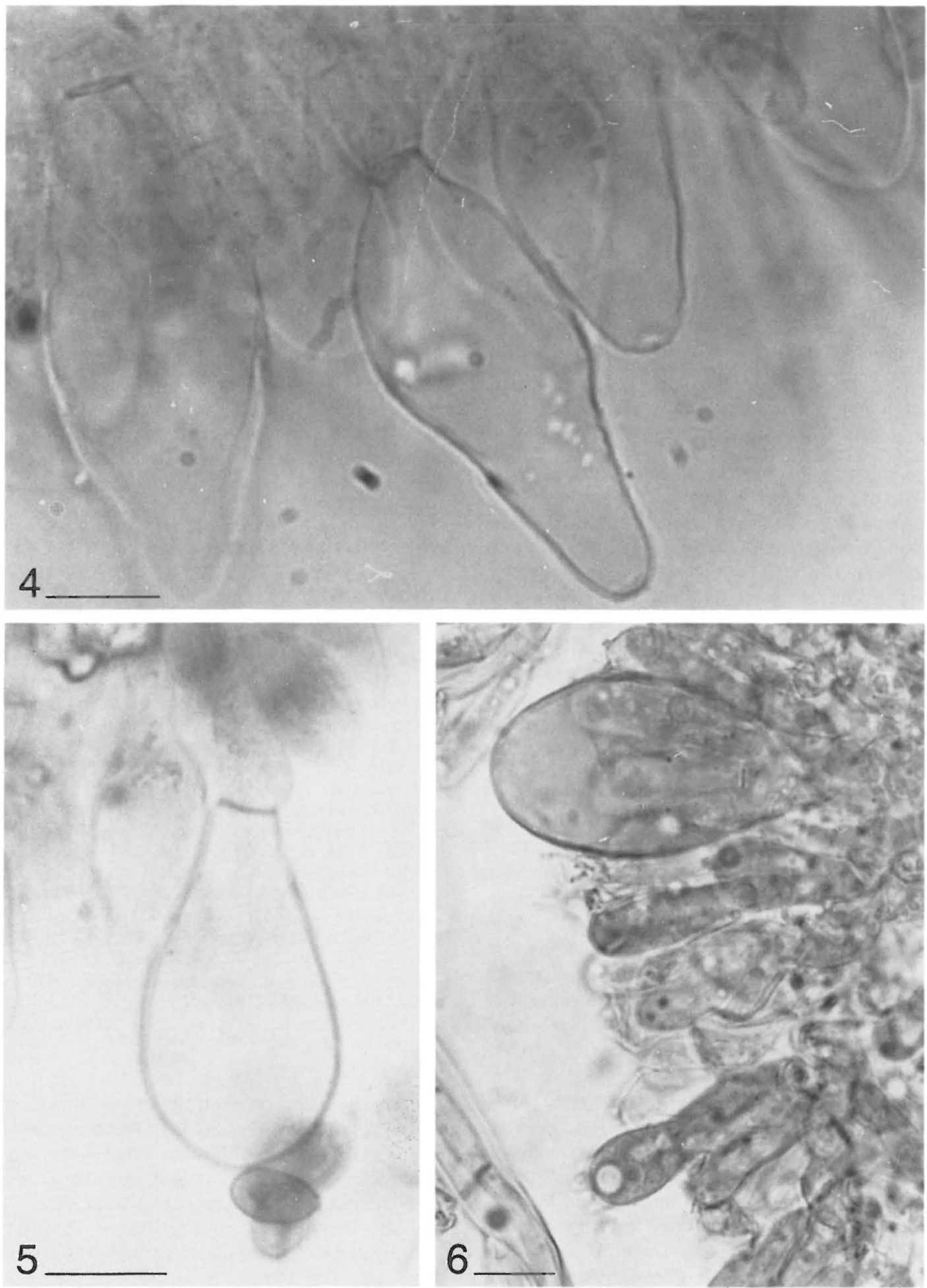

Figs. 4-6. Microscopic details of $T$. singidensis. Bar $=10 \mu \mathrm{m} .-4$ : Cheilocystidia (type). -5 : Cheilocystidium (1125). - 6: Pleurocystidium and basidia (1130). 
Termitomyces singidensis Saarim. \& Härk., sp. nov. - Figs. 1-9

Pileus 4-8(-10) $\mathrm{cm}$ in diametro, planoconvexus, umbonatus, brunneus, primum velo crasso tectus, deinde pruinosus et squamulosus. Lamellae liberae, annexae vel sinuatae, crebrae, tenues, albae vel ravido-albae. Stipes super humum aequicrassus, albus, infra humum primum interdum paolo expansus, deinde attenuatus et pseudorrhizam longam formans. Sporae ellipsoideae, inamyloideae, 5.9- $\underline{6.57}-8.5$ $\times 3.5-4.06-5 \mu \mathrm{m}$. Squamae superficiei pilei sphaerocystis semiglobularibus, 9-60 × 7-49 $\mu \mathrm{m}$.

Type: Tanzania. Central Prov.: Singida Distr., SW of Singida, Mtamaa village (04 34 DC), degraded bushland, 1500 m, 16.XII.1991 Saarimäki et al. 1129 (H, holotype).

Pileus 4-8(-10) $\mathrm{cm}$ in diameter, convex to flat, usually with a small, sharp-pointed, but sometimes broadly conical perforatorium. Young pileus totally covered with a whitish, thick and fluffy veil which remains on the brown, matted surface as a pruinose and squamulose patch around the disc, and with concentrically arranged fugacious, pustulate squamules; often bearing soil fragments. Margin entire or cracked, sometimes appendiculate and overhanging the gills. Pellicle thick. Lamellae free to adnexed or sinuate, crowded, thin, white to greyish white, up to $10 \mathrm{~mm}$ broad, margins entire. Stipe central, cylindric above ground level, (5-)11-28 $\mathrm{mm}$ in diameter, white, smooth, solid, fibrous, sometimes slightly expanded below ground level before tapering to a long, white pseudorrhiza. Sometimes veil remnants form a superior, membranous annulus around the stipe. Context in pileus white, firm, up to $12 \mathrm{~mm}$ thick; hyphae hyaline, thin-walled, 3-8(-15) $\mu \mathrm{m}$ in diameter; laticiferous hyphae present; in stipe white, fibrous. Smell faint and rubber-like. Taste mild, pleasant. Spore print pinkish cream. Spores ellipsoid to broadly ellipsoid, smooth, thinwalled, with some guttules, inamyloid, 5.9$\underline{6.57}-8.5 \times 3.5-\underline{4.06}-5 \mu \mathrm{m}, \mathrm{Q}=1.54-1.69$. Basidia clavate, 23-31 x 6.0-9.0 $\mu \mathrm{m}$, with four sterigmata. Lamella edge fertile or sterile, crowded with cheilocystidia. Cheilocystidia hyaline, variable in shape, pyriform, broadly clavate, ventricose or utriform, often with a pedicellate base, 20-75 x 10-27 $\mu \mathrm{m}$. Pleurocystidia similar to the cheilocystidia or greatly inflated, 33-70 x 12-35 $\mu \mathrm{m}$. Hymenophoral trama subparallel, hyphae hyaline, thin-walled, $3-5 \mu \mathrm{m}$ in diameter, often inflated, and then up to $22 \mu \mathrm{m}$ in diameter. Laticiferous hyphae present. Subhymenium pseudoparenchymatous. Pileal surface consists of repent, subparallel, hyaline, septate hyphae, 3-5 $\mu \mathrm{m}$ in diameter. Velar squamules on pileus surface composed of an aggregation of subglobose sphaerocysts, 9-60 x 7-49 $\mu \mathrm{m}$. The annulus consists of similar sphaerocysts. All hyphae lack clamp connections.

Etymology: The epithet singidensis refers to Singida, the collection site of the type material in Tanzania.

This species is easily recognizable by its whitish, woolly velar remnants over the brown surface of the pileus. They form a distinct, more or less squamulose patch around the small, sharppointed perforatorium and concentric rings of fugacious squamules elsewhere. Sometimes these squamules are so abundant that the pileus resembles that of a species of Amanita Pers.

\section{Specimens examined}

Tanzania. Southern Highlands Prov.: Iringa Distr., Nyololo village (08 35 CA), in field, 1900 m, 14.XII.1990 Saarimäki et al. 630. Central Prov.: Singida Distr., $60 \mathrm{~km}$ NW of Singida, along the road to Nzega, Misigiri village (04 34 DA), in miombo woodland, $1500 \mathrm{~m}, 15 . X I I .1991$ Saarimäki et al. 1123; $50 \mathrm{~km} \mathrm{NW}$ of Singida, along the road to Nzega, Mgundu village (04 34 DA), in degraded miombo woodland, $1500 \mathrm{~m}, 15$.XII.1991 Saarimäki et al. $1124 ; 8 \mathrm{~km}$ SW of Singida, Mwamkoko village (04 34 DA), in degraded bushland, 1500 m, 15.XII.1991 Saarimäki et al. 1125; Singida, specimen purchased in Singida market (04 34 DC), 16.XII.1991 Saarimäki et al. 1127; 5 $\mathrm{km}$ SW of Singida, near Lake Kindai, Unyanga village (04 34 DC), 1500 m, 16.XII.1991 Saarimäki et al. 1128; holotype; Singida, specimen purchased in a small market (04 34 DC), 16.XII.1991 Saarimäki et al 1130; $30 \mathrm{~km} \mathrm{~S}$ of Singida, along the road to Dodoma, Kipumbuiko village (05 $34 \mathrm{BB}$ ), in degraded scrub, $1500 \mathrm{~m}, 17 . X I I .1991$ Saarimäki et al. $1131 ; 45 \mathrm{~km} \mathrm{~S}$ of Singida, along the road to Dodoma (05 $34 \mathrm{BB}$ ), in miombo woodland, $1450 \mathrm{~m}$, 17.XII.1991 Saarimäki et al. 1132.

\section{Ecology}

Fruit bodies occurred singly or scattered in degraded miombo woodlands or scrub on sandy soil (Fig. 10). The habitats have been impoverished due to deforestation, cultivation and over-grazing. In many places Acacia scrub, a probable secondary succession of miombo woodland, exists. Termite nests were numerous in the area. The termites collected from the close vicinity of the fruit bodies have been identified as the genus Odontotermes. 

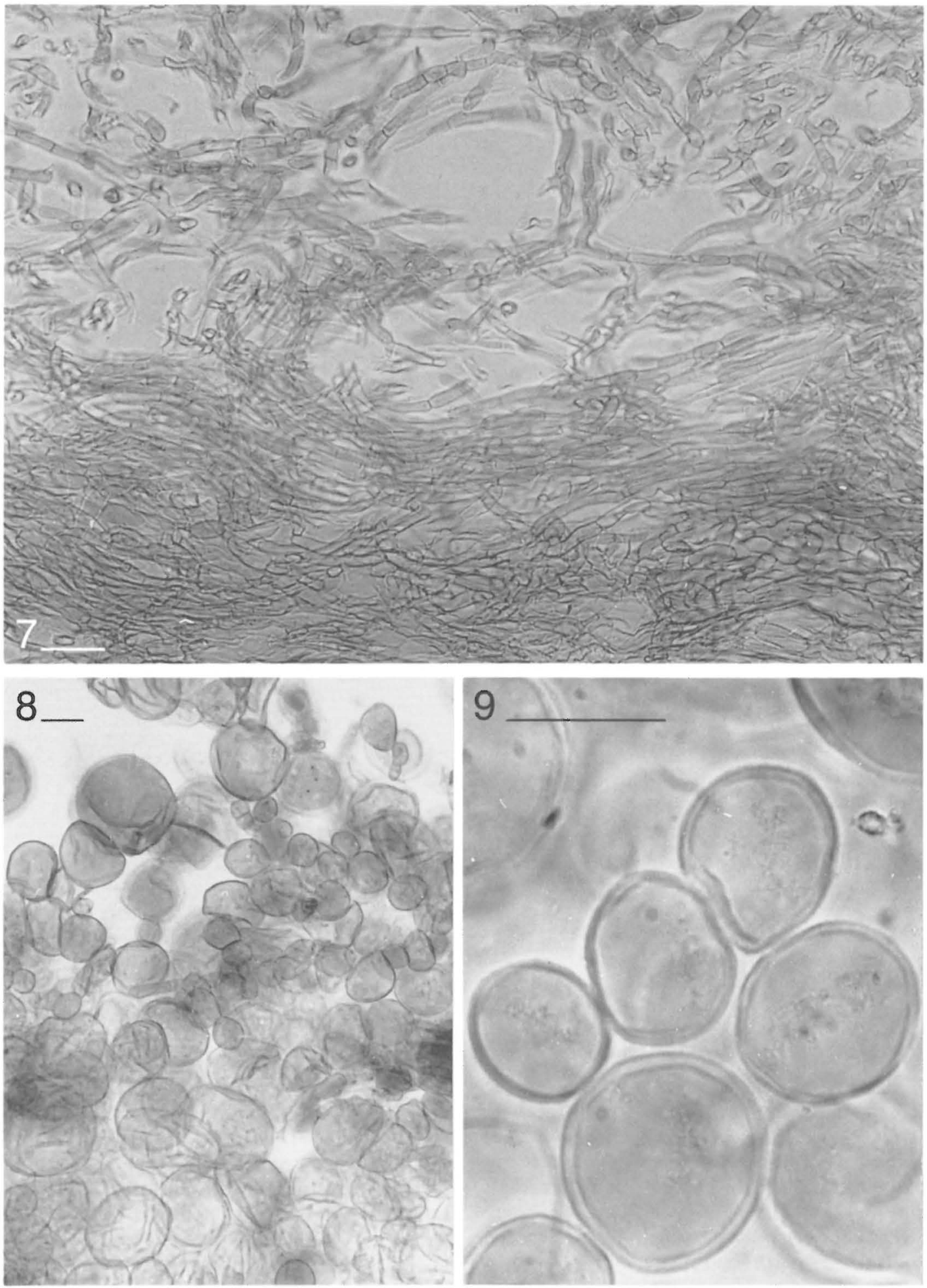

Figs. 7-9. Microscopic details of T. singidensis. Bar $=20 \mu \mathrm{m} .-7$ : Longitudinal section of pileipellis (1127). - 8: Sphaerocysts from pileal squamules (1125). — 9: Sphaerocysts from annulus (1125). 


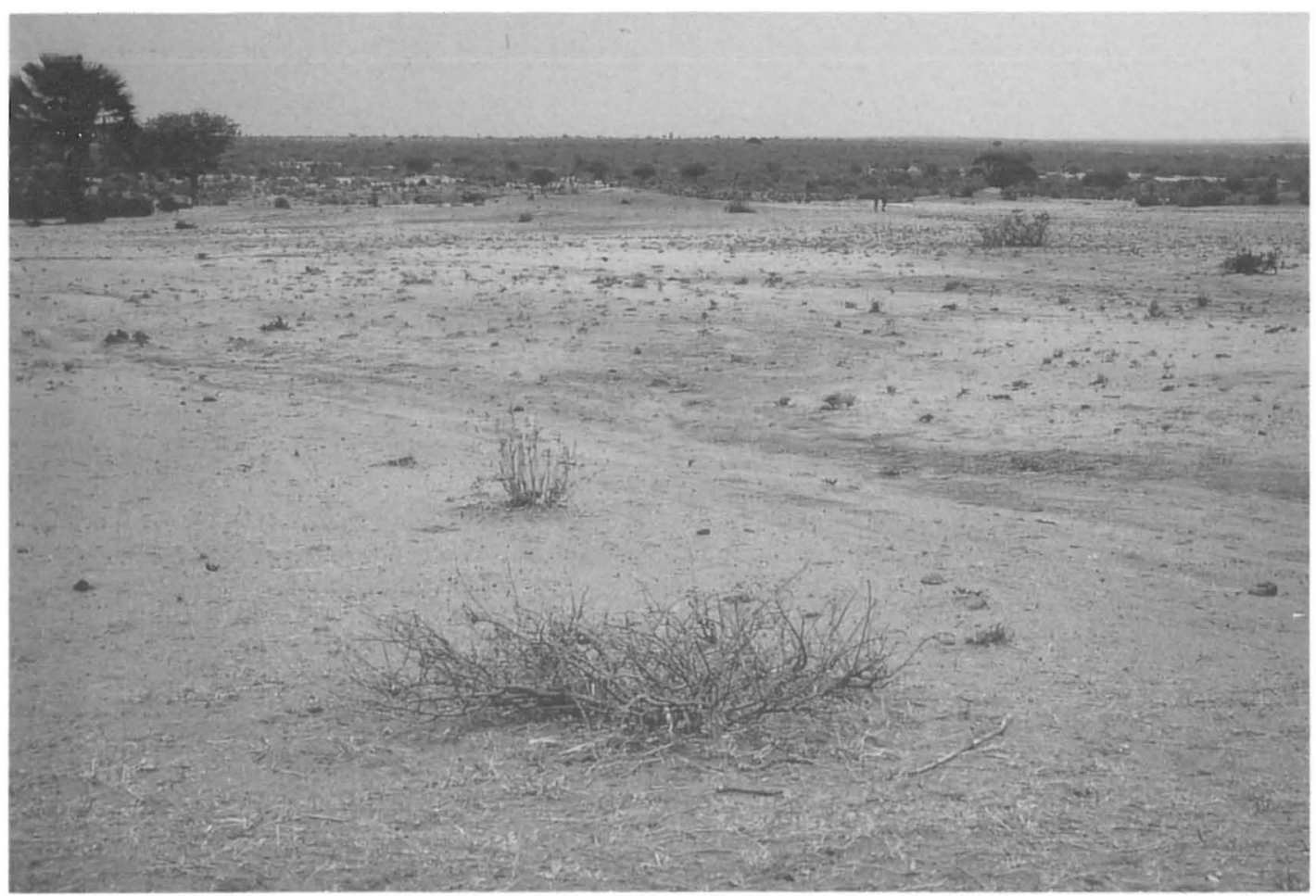

Fig. 10. Degraded and impoverished habitat of T. singidensis near Singida.

According to the local people, fruit bodies appear from October to January, if there has been enough rain.

\section{Utilization}

Termitomyces singidensis is called 'Impota' or 'Impora' by the Turu, 'Uloze' by the Iramba, and 'Witali' by the Hehe.

It is highly valued, and during the mushroom season fruit bodies are eagerly sought for food. When we asked people to estimate the deliciousness of this species on a scale from 1 to 3 , they most often gave it the highest rating. All parts, even the long pseudorrhiza, are eaten. According to the native people it is best when cooked in a stew or soup but it may even be eaten raw. When collected in great quantities the fruit bodies can be sun-dried and preserved in containers for future use.

Besides home consumption, Termitomyces singidensis is also offered for sale (Fig. 3). Women and children can earn extra income by selling mushrooms in markets and on roadsides, and usually children dig out mushrooms for their mothers. In Singida District, where this species seemed to be very common, we could see groups of small children everywhere wandering with a digging stick in one hand and in the other a string or a bag full of mushrooms (Fig. 11). The collecting of fruit bodies of $T$. singidensis and the termites associated with them can be seen in a video taken during our collecting trip to Tanzania (Härkönen \& Härkönen 1992). The habits regarding Tanzanians' use of mushrooms will be compiled from the interviews and published later. 


\section{Discussion}

Although in some features Termitomyces singidensis resembles the other known Termitomyces species $T$. lanatus R.Heim, $T$. magoyensis Otieno, T. mammiformis R.Heim and $T$. schimperi (Pat.) R.Heim, it can readily be distinguished from them in several characters. The description of T. lanatus (Heim 1977) includes only macroscopic characters, and the original material was not found in the collections of the Museum of Natural History of Paris (PC) despite a long search. Moreover, the T. lanatus description was invalid because the type was not indicated (Code Art. 37.1). The description of $T$. magoyensis is given by Otieno (1964). For the other two species, detailed and illustrated works are by Heim (1977) and Pegler (1977). Van der Westhuizen \& Eicker $(1990,1991)$ have focused special attention on $T$. schimperi from South Africa and Namibia. In addition, Saarimäki has studied an original collection of T. mammiformis (Moyen-Congo français: palmeraie d'Etoumbi et environs, termitiéres souterraines, II.1948 $R$. Heim R5-R15).

Initially the pileus of Termitomyces lanatus is covered by a thick greyish veil (Heim 1977). Later it becomes marked with large blackish brown spots and farinose squamules partially covering a whitish pileipellis, whereas the velar remains of $T$. singidensis form whitish squamules over the brown pileipellis. The form of the perforatorium differs between these two species in being broad, slightly pronounced, blunt and smooth in T. lanatus, but mostly small and sharppointed in $T$. singidensis. In distinction to the white pseudorrhiza of $T$. singidensis the subterranean pseudorrhiza of $T$. lanatus is dark brown.

The other three species mentioned above have pustulate velar squamules on the pileus. Termitomyces mammiformis is about the same size as $T$. singidensis but always has a prominent, well-differentiated, and scrobiculate perforatorium (Heim 1977, Pegler 1977). T. schimperi and T. magoyensis are much larger, and differ microscopically notably in their septate cystidia. $T$. schimperi lacks a conical or mucronate perforatorium (Pegler 1977, van der Westhuizen \& Eicker 1990, 1991). As for T. magoyensis, Otieno (1964) states that its pileus has no visible perforatorium when expanded, but in another

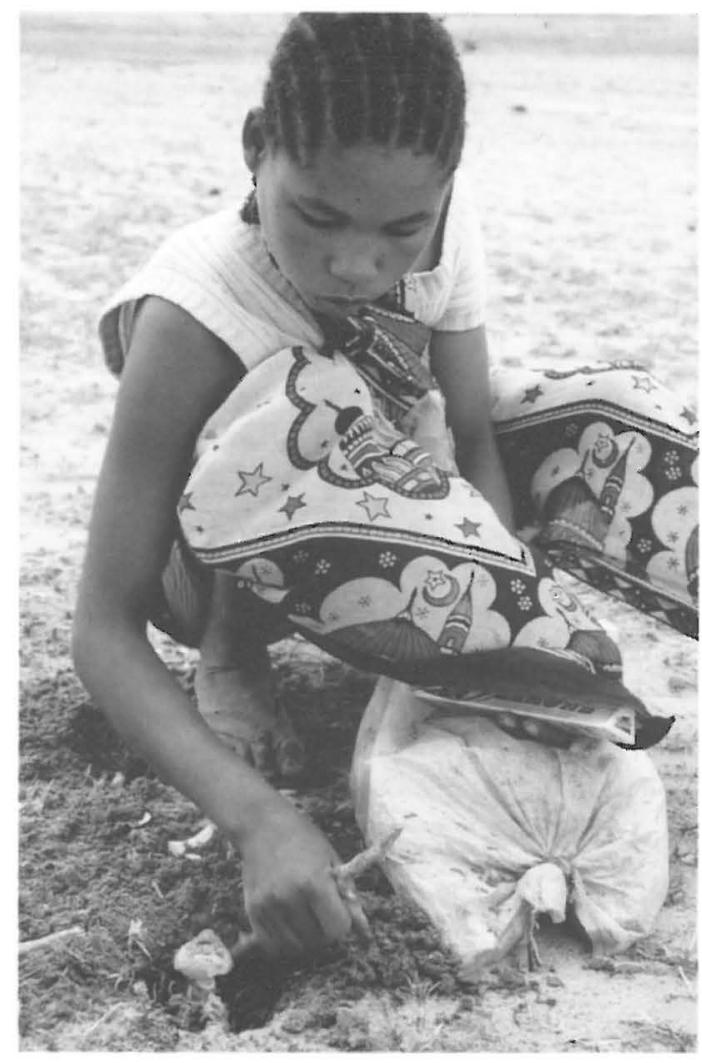

Fig. 11. A Nyaturu girl digging up T. singidensis for her mother. Selling a sackful of fruit bodies at the market earns a family additional income.

description he (Otieno 1969) describes $T$. magoyensis as having a broad, dark umbo. Microscopically $T$. singidensis is closest to $T$. mammiformis.

Acknowledgements. We wish to thank the curator of the Museum of Natural History of Paris (PC) for arranging loans, Heino Vänskä, Lic. Phil. (Helsinki) for preparing the Latin description, and Dr. M.A. Njau (Dar es Salaam) for identifying the termites collected with the fruit bodies. Prof. Tuomo Niemelä gave us valuable advice and Dr. Carol Norris revised the English of the manuscript. The Finnish International Developmental Agency (FINNIDA) financed the research. 


\section{References}

Härkönen, M. 1992: Wild mushrooms, a delicacy in Tanzania. - Univ. Helsingiensis 12:29-31.

Härkönen, M. \& Härkönen, K. 1992: Edible mushrooms of Tanzania. A video tape. - University of Helsinki, Department of Botany and Audio-Visual Centre: 55 min.

Härkönen, M., Saarimäki, T. \& Mwasumbi, L. 1993a: Setting up a research project on Tanzanian mushrooms and their uses. - Proc. XIII AETFAT Congress, Zomba, Malawi (in press).

Härkönen, M., Saarimäki, T., Mwasumbi, L. \& Niemelä, T. 1993b: Collection of the Tanzanian mushroom heritage as a form of developmental cooperation between the universities of Helsinki and Dar es Salaam. - Aquilo Ser. Bot. 31:99-105.

Härkönen, M., Buyck, B., Saarimäki, T. \& Mwasumbi, L. 1993c: Tanzanian mushrooms and their uses 1 . Russula. - Karstenia 33:16-50.

Härkönen, M., Saarimäki, T. \& Mwasumbi, L. 1993d: Tanzanian mushrooms and their uses 2. An edible species of Coprinus section Lanatuli. - Karstenia 33:51-59.

Heim, R. 1977: Termites et champignons. - 205 pp. Boubée, Paris.

Leistner, O.A. \& Morris, J.W. 1976: Southern African Place Names. - Annals Cape Prov. Mus. 12:1-565.
Morris, B. 1986: Notes on the genus Termitomyces Heim in Malawi. - Soc. Malawi J. 39:40-49.

Otieno, N.C. 1964: Contributions to a knowledge of termite fungi in East Africa. - Proc. East African Acad. 2:109-121.

Otieno, N.C. 1969(1968): Further contributions to a knowledge of Termite fungi in East Africa: the genus Termitomyces Heim. - Sydowia 22:160-165.

Pegler, D.N. 1977: A preliminary agaric flora of East Africa. - Kew Bull. Add. Ser. VI:1-615.

Pegler, D.N. \& Rayner, R.W. 1969: A contribution to the agaric flora of Kenya. - Kew Bull. 23:347-412.

Piearce, G.D. 1987: The genus Termitomyces in Zambia. - The Mycologist 1:111-116.

Polhill, D. 1988: Flora of tropical East Africa. Index of collecting localities. - 398 pp. Royal Bot. Gardens, Kew.

Singer, R. 1986: The Agaricales in modern taxonomy. 981 pp. Koeltz, Koenigstein.

Van der Westhuizen, G.C.A. \& Eicker, A. 1990: Species of Termitomyces occurring in South Africa. - Mycol. Res. 94:923-937.

Van der Westhuizen, G.C.A. \& Eicker, A. 1991: The 'Omajowa' or 'Termitenpilz', Termitomyces sp. (Agaricales) of Namibia. — S. African J. Bot. 57:6770.

Received on 3 November 1993 